\title{
Enhanced p62 expression triggers concomitant autophagy and apoptosis in a rat chronic spinal cord compression model
}

\author{
ZHI CHEN $^{1 *}$, QINGGE FU $^{2 *}$, BAOLIANG SHEN $^{3 *}$, XUAN HUANG $^{1}$, KUN WANG $^{1}$, \\ PING HE ${ }^{1}$, FENGNING LI ${ }^{1}$, FAN ZHANG ${ }^{1}$ and HONGXING SHEN ${ }^{1}$ \\ ${ }^{1}$ Department of Orthopaedics, Changhai Hospital Affiliated to The Second Military Medical University, Shanghai 200433; \\ ${ }^{2}$ Department of Emergency and Trauma, Shanghai East Hospital, Tongji University School of Medicine, Shanghai 200120; \\ ${ }^{3}$ Department of Orthopaedics, Shanghai Jiading Central Hospital, Shanghai 201800, P.R. China
}

Received November 6, 2013; Accepted March 10, 2014

DOI: $10.3892 / \mathrm{mmr} .2014 .2124$

\begin{abstract}
Chronic spinal cord compression is the result of mechanical pressure on the spinal cord, which in contrast to traumatic spinal cord injury, leads to slowly progressing nerve degeneration. These two types of spinal cord injuries may trigger similar mechanisms, including motoric nerve cell apoptosis and autophagy, however, depending on differences in the underlying injury severity, nerve reactions may predominantly involve the conservation of function or the initiation of functions for the removal of irreversibly damaged cells. p62 is a multidomain adapter protein, which is involved in apoptosis and cell survival as well as autophagy, and is a common component of protein aggregations in neurodegenerative diseases. In the present study, a rat chronic spinal cord compression model was used, in which the spinal cord was progressively compressed for six weeks and then constantly compressed for another 10 weeks. As a result Basso, Beattie and Bresnahan locomotor scaling revealed a gradual score decrease until the 6 th week followed by constant recovery until the 16th week after spinal cord compression was initiated. During the first eight weeks of the experiment, p62 and nuclear factor $-\kappa \mathrm{B}(\mathrm{NF}-\kappa \mathrm{B})$ were increasingly expressed up to a constant plateau at 12-16 weeks, whereas caspase 3 exhibited a marginally enhanced expression at 8 weeks, however, reached a constant maximum peak 12-16 weeks after the beginning of spinal cord compression. It was hypothesized that, in the initial phase of spinal cord compression, enhanced p62 expression triggered $\mathrm{NF}-\kappa \mathrm{B}$ activity, directing the cell responses
\end{abstract}

Correspondence to: Dr Hongxing Shen, Department of Orthopaedics, Changhai Hospital Affiliated to The Second Military Medical University, 168 Changhai Road, Shanghai 200433, P.R. China

E-mail: shenhxgk@126.com

*Contributed equally

Key words: p62, chronic spinal cord compression model, cell autophagy, cell apoptosis mainly to cell survival and autophagy, whereas following eight weeks of spinal cord compression, caspase 3 was additionally activated indicating cumulative elimination of irreversibly damaged nerve cells with highly activated autophagy.

\section{Introduction}

Chronic spinal cord injury, including cervical spondylotic myelopathy (CSM), is the most common spinal cord disorder in elderly individuals and is caused by degenerative alterations in the spine, including degeneration of joints, intervertebral discs, ligaments and connective tissue of the cervical vertebrae with concomitant spinal canal stenosis (1). Canal dimensions were reported to be determinants of symptom production and neurological compromises $(2,3)$. The gray matter in the compressed spine segments becomes atrophic with thin myelinated fibers, while denuded axons occur in the damaged white matter (4). As a result of compression, the affected area of the spinal cord may become ischemic $(5,6)$ or hypoxic $(7)$ and, besides mechanical observations, other studies have focused on chronic spinal cord compression-associated neural apoptosis $(8-10)$, in particular of oligodendrocytes $(11,12)$, which is considered to be the result of compression-associated ischemia. As an alternative to apoptosis, autophagy serves as a repair mechanism for damaged cell organelles as well as for the clearance of encapsulated proteins and has an important physiological cytoprotective function against neurodegenerative diseases (13-17). Following spinal cord injury, the major mechanism triggered is apoptosis (18), which has been verified by Fas death receptor pathway inactivation experiments $(19,20)$ showing reduced apoptosis of neuron cells following spinal cord injuries. Other studies have demonstrated that rapamycin (an mTOR inhibitor) was also able to enhance the survival rate of neuron cells following spinal cord injury via induction of autophagy $(21,22)$. Studies with genetically modified mice demonstrated that protein 62/sequestosome 1 (p62/SQSTM1) is important in a number of cellular functions, including bone remodeling (23), obesity (24) and cancer (25). For neurons, several studies have described p62 as a common component of protein aggregates that were identified in protein aggregation diseases affecting the brain, including Lewy bodies in Parkinson's disease, huntingtin aggregates 
in Huntington's disease and neurofibrillary tangles in Alzheimer's disease (26-28). p62 is a nucleoporin, which binds ubiquitinated proteins via its ubiquitin-associated domain and microtubule-associated protein 1A/1B light chain 3 (LC3) via its LC3-interacting region, thereby acting as an adaptor between the autophagic machinery and misfolded proteins (29). Additionally, p62 binds with polyubiquitinated caspase 8 and triggers programmed cell death with aggregation into nuclear speckles (30). The p62 protein also contains a tumor necrosis factor receptor associated factor 6 (TRAF6)-binding domain and, following binding and subsequent activation, TRAF6 induces the nuclear factor $-\kappa \mathrm{B}(\mathrm{NF}-\kappa \mathrm{B})$ pathway $(31,32)$. Since activated NF- $\kappa \mathrm{B}$ leads to a reduction in apoptosis, the p62 nuclear speckles may be signaling hubs that determine whether cells survive, by triggering the $\mathrm{NF}-\kappa \mathrm{B}$ pathway, or die, by activating downstream effector caspases in spinal cord injuries (33). The present study investigated the role of p62 in chronic spinal cord injury using a rat model of chronic spinal cord compression.

\section{Materials and methods}

Chronic spinal cord injury rat model. A chronic spinal cord compression rat model was employed (34) with slight modifications. A total of 48 female Sprague-Dawley rats, weighing 170-200 g with an average weight of $185 \mathrm{~g}$ (Charles River Laboratories, Inc., Wilmington, MA, USA) were divided into two groups: the sham group (no compression; $n=6$ ) and the compression group (compression with a screw advanced by $0.3 \mathrm{~mm}$ per week; one turn; $\mathrm{n}=42$ ) as indicated (Figs. 1 and 2). All the rats were intraperitoneally anesthetized with $10 \mathrm{mg} / \mathrm{kg}$ xylazine (Rompun; Bayer, Istanbul, Turkey) and $50 \mathrm{mg} / \mathrm{kg}$ ketamine hydrochloride (Ketalar; Pfizer, Istanbul, Turkey). Progressive cord compression was achieved at the T10 level by sequential turning of a threaded screw (diameter $2 \mathrm{~mm}$; one turn $=0.3 \mathrm{~mm}$ ). The compression was performed precisely using a microscope at an oblique angle (World Precision Instruments, Inc., Sarasota, FL, USA). The sham-surgery animals underwent identical surgical procedures as above with the exception of cord compression. Acute post-surgical care included rehydration with $5 \mathrm{ml}$ of sterile saline (Baxter, Mississauga, Ontario, Canada), increasing body temperature with a focused heat lamp, warm towels and subcutaneous injection of buprenorphine hydrochloride $(0.05 \mathrm{mg} / \mathrm{kg})$ immediately following surgery in order to minimize post-surgical pain. Post-surgical care also included individually housing the animals in a room with ambient temperature and controlled light conditions. All animals were sacrificed at different weeks following surgery. All experimental procedures were performed in compliance with the Guidelines for the Care and Use of Animals described in the Guidelines established by the Institute of Laboratory Animal Sciences, Faculty of Medicine of the Second Military Medical University (Shanghai, China). The present study was approved by the ethics committee of Changhai Hospital (Shanghai, China).

Basso, Beattie and Bresnahan locomotor (BBB) score and assessment. Behavioral functional deficits due to chronic compression were characterized weekly using a variety of neurobehavioral tests, including the BBB locomotor scale rating until 16 weeks post-surgery. Observers blinded to the compression group evaluated all the scores. Locomotor deficits were assessed by two independent observers using the BBB locomotor rating scale (35). The 22-point (0-21) scale included monitoring of hindlimb movements, trunk position and stability, stepping, co-ordination, paw placement, toe clearance and tail position, while the rats were placed individually in an open field with a non-slippery surface. Each rat was observed for a period of $4 \mathrm{~min}$ at each testing session and the scores were averaged across the right and left limbs to derive a final motor recovery score for each week of testing. A score of 21 indicated unimpaired locomotion as observed in the control rats.

Tissue preparation. For immunohistochemical analyses, the rat spines were perfused with normal saline under intraperitoneal anesthesia and then fixed with $4 \%$ paraformaldehyde (Cell Chip Biotechnology Co., Ltd., Beijing, China). Then, the spinal cords were embedded in paraffin and $4-\mu \mathrm{m}$ transverse slides were prepared from areas, which were located $2 \mathrm{~mm}$ caudal or cranial of the T10 screw compression site. For western blot analyses, the $10-\mathrm{cm}$ rat cervical spinal cords ( $n=3$ per group) were dissected and homogenized in tissue protein extraction reagent (Pierce Protein Research Products, Rockford, IL, USA).

Histological analysis. The sections prepared from treated and non-treated rats were fixed with $4 \%$ paraformaldehyde (Maya-Reagent Co., Ltd., Jiaxing, Zhejiang, China) and stained with hematoxylin and eosin (Beyotime Institute of Biotechnology Co., Ltd., Shanghai, China).

Western blot analyses. For western blot analyses, $\sim 30 \mu \mathrm{g}$ protein was loaded onto 8\% NuPAGE precast gels (NP0322; Invitrogen Life Technologies, Carlsbad, CA, USA) and transferred onto nitrocellulose membranes. The membranes were inhibited in Tris-buffered saline/Tween 20 buffer pH 7.5, containing 0.15 M NaCl (313-20; Beijing Biotechnology Co., Ltd., Beijing, China) and 0.05\% Tween 20 (93773; Sigma-Aldrich, Shanghai, China) containing 3\% milk powder for $1 \mathrm{~h}$ at room temperature. The membrane was incubated with the primary antibody overnight at $4^{\circ} \mathrm{C}$, followed by secondary horseradish peroxidase (HRP)-labeled antibody incubation for $1 \mathrm{~h}$ at room temperature. Detection was performed using the ECL detection system (Millipore, Darmstadt, Germany) and protein bands were visualized using an ImageQuant digital imaging system (ChemiQ 4600, Shanghai, China).

Immunohistochemistry. For immunohistochemistry, the sections were treated with xylene for $10 \mathrm{~min}$, alcohol hydration for $15 \mathrm{~min}$ and methanol containing 3\% hydrogen peroxide for $10 \mathrm{~min}$, and were incubated at a high temperature and pressure for $2 \mathrm{~min}$. The sections were then washed in phosphate-buffered saline containing $0.1 \%$ Triton X-100 (Bio Basic Inc., Toronto, Canada) for $5 \mathrm{~min}$, blocked with $5 \%$ bovine serum albumin for $1 \mathrm{~h}$ at room temperature and then incubated with the primary antibody overnight at $4^{\circ} \mathrm{C}$. The following primary antibodies were used: rabbit anti-p62 (ab91526; Abcam, Cambridge, UK), mouse anti-NF-кB protein (4764s; Santa Cruz, Dallas, TX, USA), mouse 


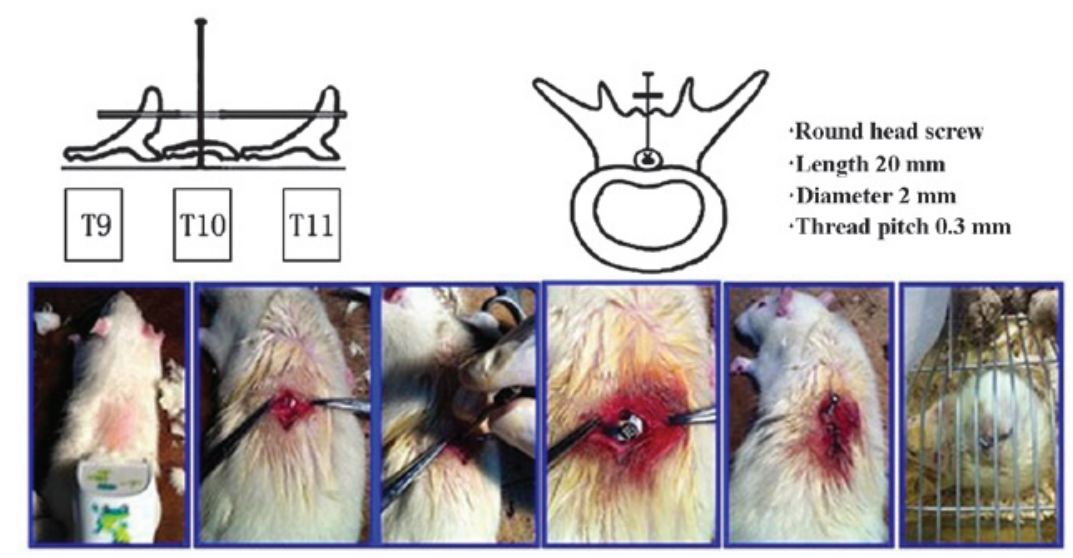

Figure 1. Images of the screw insertion for establishing progressive cord compression (diameter $2 \mathrm{~mm}$ ).
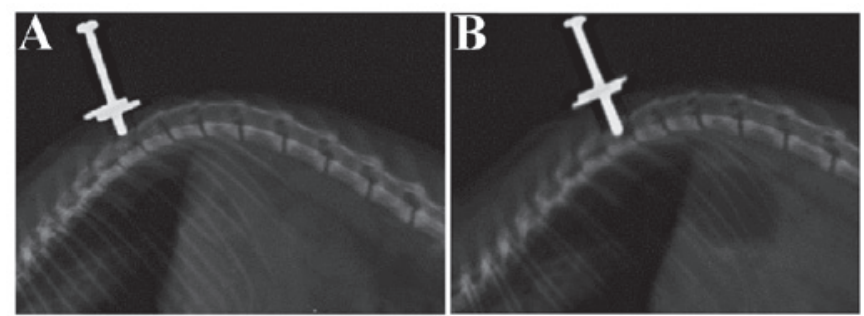

Figure 2. X-ray images of the rat spine with advanced screw compression of the spinal cords. (A and B) $1.8 \mathrm{~mm}$ depth until week 6 post-surgery.

anti-caspase-3 (9662s; Santa Cruz) and rabbit $\beta$-actin (4967; Cell Signaling Technology, Boston, MA, USA). The following secondary antibodies were used: anti-rabbit HRP-linked antibody (7074; Cell Signaling Technology) and anti-rat HRP-linked antibody (7077; Cell Signaling Technology). Finally, the sections were incubated with peroxidase-labeled polymer-HRP conjugated antibodies (HRP conjugated anti-rabbit IgG (Cell Signaling Technology) and HRP conjugated anti-rat IgG (Cell Signaling Technology) for $1 \mathrm{~h}$ at room temperature and then visualized with $0.02 \%$ diaminobenzidine (ZSGB-Bio Co., Ltd., Beijing, China). The sections were examined with a microscope (Leica TCS SP2; Leica, Solms, Germany) and the images were captured using an image analysis system Lumina Vision (Mitani Corp., Fukui, Japan).

Quantitative PCR ( $q P C R)$. Total RNA was extracted from spinal cord tissues using TRIzol ${ }^{\circledR}$ reagent (Invitrogen Life Technologies, Grand Island, NY, USA) and concentrations were evaluated using a Shimadzu UV-1700 spectrophotometer (Shimadzu Corporation, Kyoto, Japan) at A260 nm. From each mRNA, $1 \mu \mathrm{g}$ was reverse transcribed into first-strand cDNA by SuperScript ${ }^{\mathrm{TM}}$ III Reverse Transcriptase (Invitrogen Life Technologies) in a total reaction volume of $20 \mu \mathrm{l}$. qPCR amplifications were performed in triplicate using 2X FastStart Universal SYBR-Green Master mixtures (Roche Applied Science, Mannheim, Germany) with $2 \mu \mathrm{l}$ of cDNA samples and specific primers. The primers used were as follows: p62, forward 5'-TCCCTGTCAAGCAGTATCC-3' and reverse 5'-TCCTCCTTGGCTTTGTCTC-3'; NF-кB, forward 5'-GCACCAAGACCGAAGCAAT-3' and reverse 5'-CTCCCGTAACCGCGTAGTC-3'; caspase-3, forward 5'-CAAGGGACGGGTCATGGTTC-3' and reverse 5'-AAGTGGCGTCCAGGGAGAAG-3'; LC3, forward 5'-GACT TCCGGAAAGCTCTGCT-3' and reverse 5'-ACCAGCATCGTAGAGGGTCT-3'; GAPDH, forward 5'-AATGCATCCTGCACCACCAA-3' and reverse 5'-GATGGCATGGACTGTGGTCA-3'. For each quantification, 35 PCR cycles were performed at $95^{\circ} \mathrm{C}$ for $15 \mathrm{sec}$ and $60^{\circ} \mathrm{C}$ for 1 min and monitored using an ABI Prism 7300 instrument (Applied Biosystems, Foster City, CA, USA).

Statistical analysis. The data are presented as the mean \pm standard error of the mean. Statistical analyses were performed using SigmaStat software (SPSS, Inc., Chicago, IL, USA). Analyses of neuron quantification and neurobehavioral studies (BBB tests) were performed using two-way analysis of variance (ANOVA) followed by post-hoc pair-wise multiple comparisons. A two-way ANOVA was performed to identify the differences between groups (sham versus compressed) and time. The Bonferroni test was used for post-hoc analysis to correct for multiple comparisons. $\mathrm{P}<0.05$ was considered to indicate a statistically significant difference.

\section{Results}

Establishment of a chronic progressive cervical spinal cord compression rat model. In the present study, chronic spinal cord compression was established using a threaded screw. $\mathrm{X}$-ray imaging demonstrated that the compression was $0 \%$ prior to screwing (Fig. 2A) and was increasingly induced by advancing the screw $0.3 \mathrm{~mm}$ each weak until the 6 th week after initial screw implantation (Fig. 2B). 


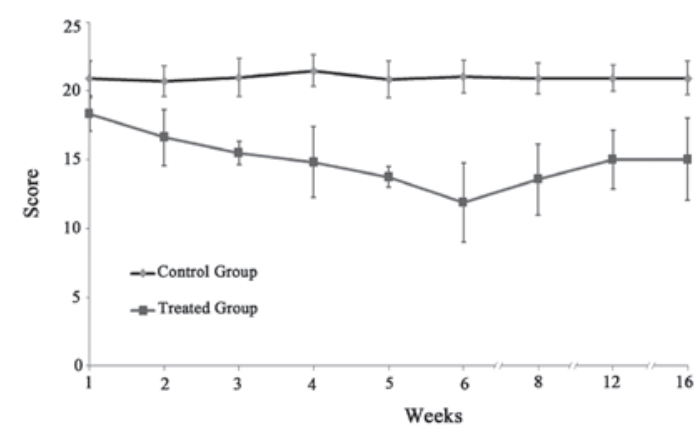

Figure 3. Basso, Beattie and Bresnahan locomotor scores of treated and control rats. $\diamond$ indicates the scores of the control and $\square$ indicates the scores of the treated animals during progressive (1st-6th week) and constant (7th-16th week) spine compression.

BBB scores 1 week after surgery were unaltered with values of $18 \pm 3$. At 2 and 3 weeks until 6 weeks (second, third, up to sixth compression) all animals demonstrated progressive decreases in BBB scores. From 6-16 weeks, the animals showed progressive improvements in nerve functionality scores. At 16 weeks post-surgery, compression animals had scores of $15.8 \pm 0.34$ (Fig. 3).

Alterations in motor neuron morphology upon compression. In the compression group, particularly in the posterior horn cell layer, large motor neurons characterized by a spindle-shape became smaller, (arrows Fig. 4B and C) and certain neurons exhibited an elongated spindle-shape (arrows Fig. 4D and E). However, the shape of the neurons returned to normal following 16 weeks (arrows Fig. 4F).

Expression of p62, NF- $\kappa B$ and caspase 3 in the compressed spines. Via immunohistochemical examination, it was revealed that the expression of p62 increased in the spinal gray matter of the caudal and epicenter area (Fig. 5). In the white matter, p62 spots were observed, which spread to the epicenter and to the caudal site of the compressed area (data not shown). In addition, $\mathrm{NF}-\kappa \mathrm{B}$ staining was enhanced in the compressed spine group at 4 and 12 weeks after surgery whereas caspase 3 staining was more apparent at 12 weeks (Fig. 5).

The expression of $\mathrm{p} 62, \mathrm{NF}-\kappa \mathrm{B}$ and caspase 3 was also quantitatively analyzed in the compressed spinal cord via western blot analysis and qRT-PCR analyses. The expression of p62 gradually increased from week 1 to week 12 with a constant high level from week 12 to week 16 after surgery. $\mathrm{NF}-\kappa \mathrm{B}$ followed this trend with gradual increases from week 1 to week 16, whereas caspase 3 started to increase at week 8 with constant high peaks at weeks 12 and 16 (Fig. 6). Chronic spinal cord injury led to p62 and NF- $\kappa$ B accumulation at 12-16 weeks, which was accompanied by enhanced caspase 3 expression from 8 weeks after surgery.

\section{Discussion}

Although the precise molecular mechanisms underlying the progressive loss of neuron cells, oligodendrocytes as well as demyelination in the spinal cord of chronic spinal cord compression remains uncertain, it is assumed that oxidative
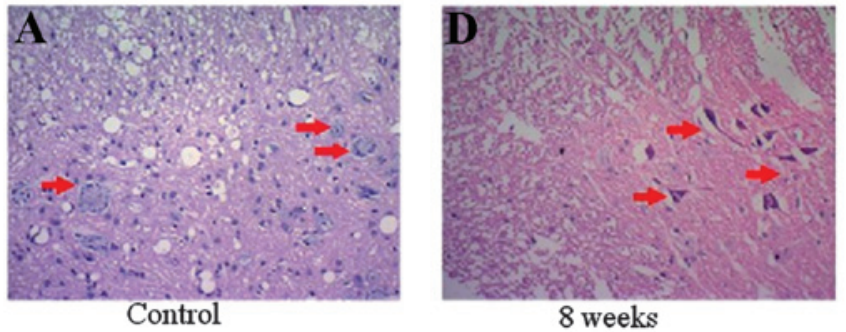

8 weeks

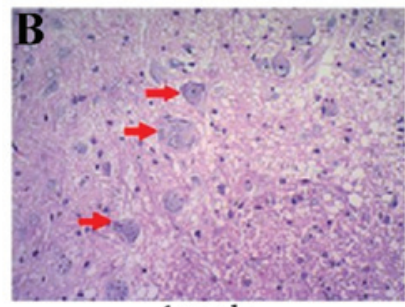

1 week

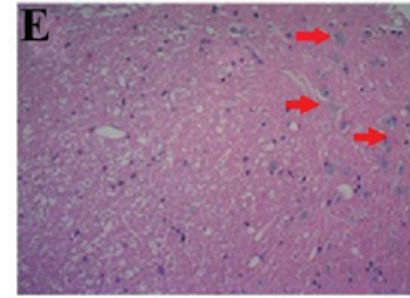

12 weeks

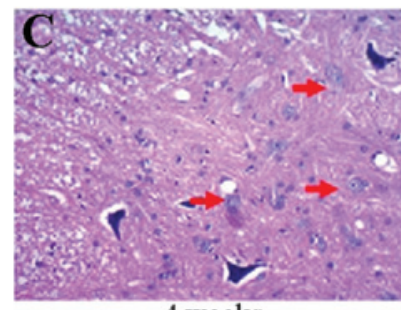

4 weeks

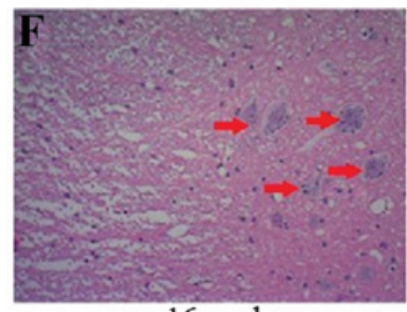

16 weeks

Figure 4. Hematoxylin and eosin staining of cervical spine slides from rats subjected to spine compression. (A) Control shows a normal cord with normal shaped motor neurons (red arrows). (B-F) Subdural and intramedullary hemorrhages as well as edema led to spindle-shaped motor neurons starting at 1 week after treatment, which recovered until 16 weeks after surgery (red arrows).

stress leads to the induction of apoptosis in injured spinal cord tissues. p62 was reported to be consistently overexpressed in the pathological neurodegenerative changes of $\mathrm{CSM}$ and is a common component of protein aggregations in neurodegenerative diseases causing neuronal loss as well as axon degeneration in the brain $(26,36)$. Accumulation of p62 without autophagy leads to liver damage due to activation of the caspase pathway $(29,30)$. However, accumulation of p62 can result in cell survival and cell death mechanisms, since $\mathrm{NF}-\kappa \mathrm{B}$ is also a p62 upregulated protein (31). NF- $\kappa \mathrm{B}$ proteins comprise a family of structurally associated 'rapid-acting' transcription factors. Stress-induced activation of $\mathrm{NF}-\kappa \mathrm{B}$ leads to the secretion of inflammatory cytokines in addition to increased expression of genes that regulate cell survival and growth as well as arrested proliferation, depending on the nature of the insult and extent of the damage (37). In the present study, p62 levels continually increased with spinal cord compression rate and time, which was accompanied by a similar increase in the expression of $N F-\kappa B$. These data suggested that the initial response to spinal cord compression was autophagy, as $\mathrm{NF}-\kappa \mathrm{B}$ is considered to be an anti-apoptotic gene (38). Additionally, NF- $\kappa \mathrm{B}$ may have directed the cell activity from potentially unconditional apoptosis to autophagy (33), thereby preventing a profuse loss of neuron cells. In the later stage of spinal cord compression without further pressure enhancement, in addition to activated autophagy, caspase 3 expression was promoted in 


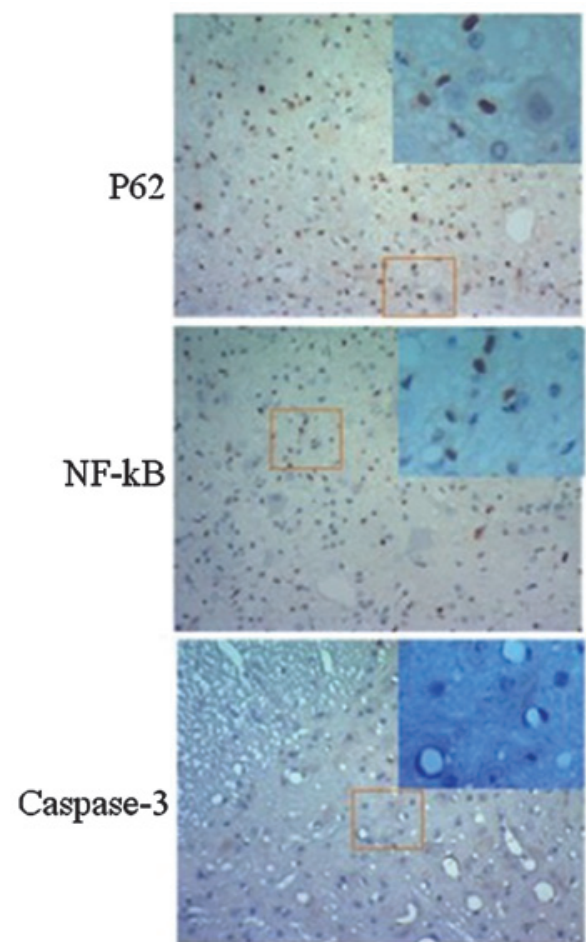

Control
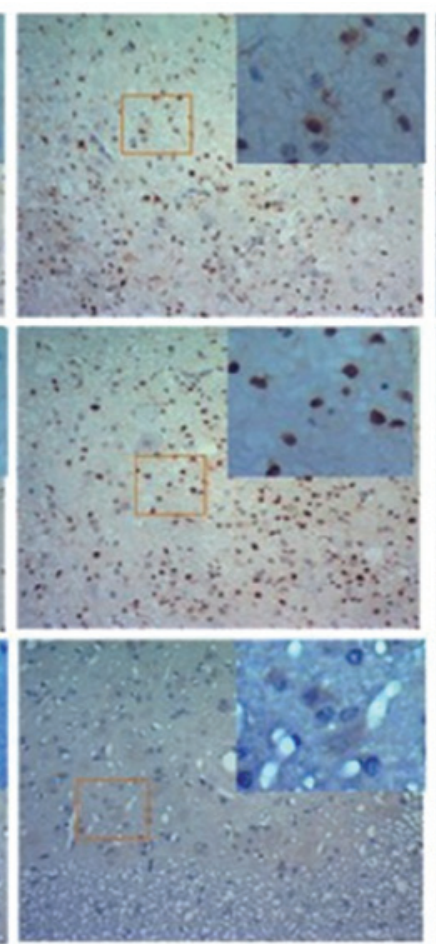

After 4 Weeks
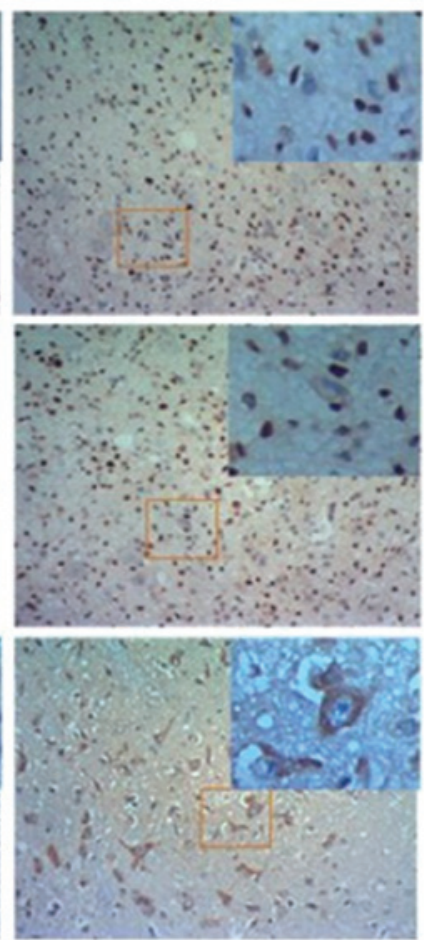

After 12 weeks

Figure 5. p62, NF-kB and caspase 3 immunostaining of rat cervical spondylotic myelopathy model spinal cord sections. The blue color indicates staining of the nuclei, whereas the brown color indicates p62, NF-кB and caspase 3 staining. The left images indicate control spine sections, in which p62, NF- $\mathrm{BB}$ and caspase 3 exhibit a low expression level. p62 and NF-кB gradually accumulated in the nuclei 4 and 12 weeks after screw insertion, whereas caspase 3 staining

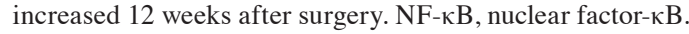

A

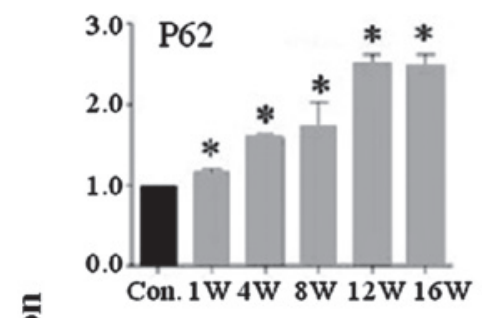

产

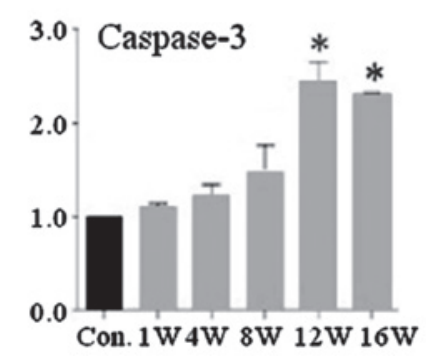

B

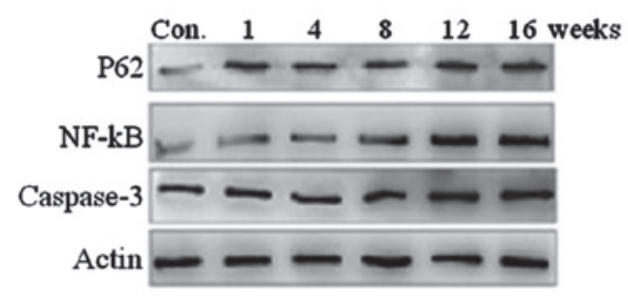

$\mathbf{C}$
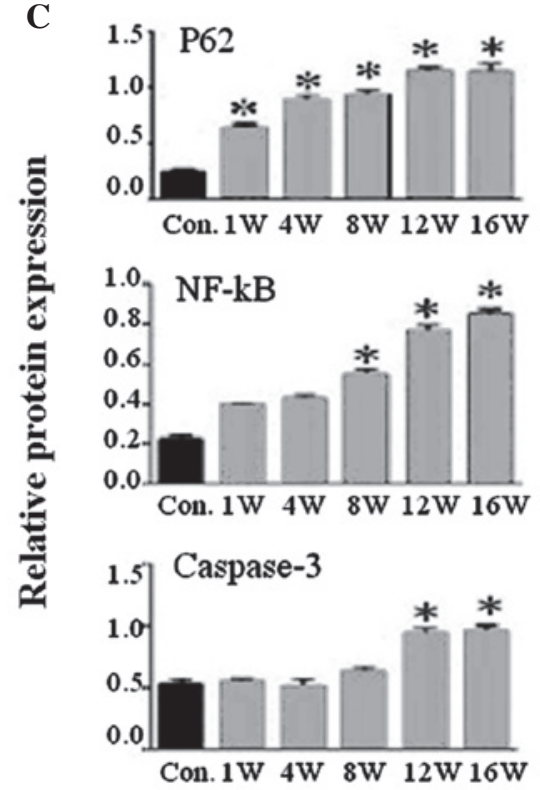

Figure 6. p62, NF-kB and caspase 3 expression in rat cervical spondylotic myelopathy model spines at the indicated time points. (A) Quantitative PCR values; (B) western blot analysis; (C) quantitative analyses of western blot data. All experiments were performed in triplicate. "P<0.05, compared with the control

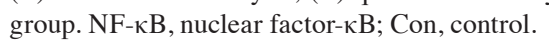


order to eliminate irreversibly damaged neuron cells. It was hypothesized that the combination of stopping screw insertion and the activation of NF- $\mathrm{KB}$ from six weeks after initial surgery led to the gradual improvements of BBB scores, and this was due to repaired cell functionality via enhanced triggered autophagy, whereas the clearance of irreparable cells was enhanced from 8 weeks after initial surgery.

\section{Acknowledgements}

This study was supported by the Shanghai Natural Science Foundation (grant no. 11JC1415600), the Scientific \& Technological Commission of Jiading District in Shanghai (grant no. 2013-KW-06), Changhai Hospital 1255 Project Funds (grant no. CH125520900) and The New One Hundred Plan in Shanghai (grant no. XBR2013099).

\section{References}

1. Hayashi H, Okada K, Hamada M, Tada K and Ueno R: Etiologic factors of myelopathy. A radiographic evaluation of the aging changes in the cervical spine. Clin Orthop Relat Res: 200-209, 1987.

2. Ogino $\mathrm{H}$, Tada $\mathrm{K}$, Okada $\mathrm{K}$, et al: Canal diameter, anteroposterior compression ratio, and spondylotic myelopathy of the cervical spine. Spine (Phila Pa 1976) 8: 1-15, 1983.

3. Edwards WC and LaRocca H: The developmental segmental sagittal diameter of the cervical spinal canal in patients with cervical spondylosis. Spine (Phila Pa 1976) 8: 20-27, 1983.

4. Ito T, Oyanagi K, Takahashi $\mathrm{H}$, Takahashi HE and Ikuta F: Cervical spondylotic myelopathy. Clinicopathologic study on the progression pattern and thin myelinated fibers of the lesions of seven patients examined during complete autopsy. Spine (Phila Pa 1976) 21: 827-833, 1996.

5. Gooding MR, Wilson CB and Hoff JT: Experimental cervical myelopathy. Effects of ischemia and compression of the canine cervical spinal cord. J Neurosurg 43: 9-17, 1975.

6. Hukuda S and Wilson CB: Experimental cervical myelopathy: effects of compression and ischemia on the canine cervical cord. J Neurosurg 37: 631-652, 1972.

7. Long HQ, Li GS, Hu Y, Wen CY and Xie WH: HIF-1alpha/VEGF signaling pathway may play a dual role in secondary pathogenesis of cervical myelopathy. Med Hypotheses 79: 82-84, 2012.

8. Fehlings MG and Skaf G: A review of the pathophysiology of cervical spondylotic myelopathy with insights for potential novel mechanisms drawn from traumatic spinal cord injury. Spine (Phila Pa 1976) 23: 2730-2737, 1998.

9. Yu WR, Baptiste DC, Liu T, Odrobina E, Stanisz GJ and Fehlings MG: Molecular mechanisms of spinal cord dysfunction and cell death in the spinal hyperostotic mouse: implications for the pathophysiology of human cervical spondylotic myelopathy. Neurobiol Dis 33: 149-163, 2009.

10. Yu WR, Liu T, Kiehl TR and Fehlings MG: Human neuropathological and animal model evidence supporting a role for Fas-mediated apoptosis and inflammation in cervical spondylotic myelopathy. Brain 134: 1277-1292, 2011.

11. Kim DH, Vaccaro AR, Henderson FC and Benzel EC: Molecular biology of cervical myelopathy and spinal cord injury: role of oligodendrocyte apoptosis. Spine J 3: 510-519, 2003.

12. Karadimas SK, Gialeli $\mathrm{CH}$, Klironomos G, et al: The role of oligodendrocytes in the molecular pathobiology and potential molecular treatment of cervical spondylotic myelopathy. Curr Med Chem 17: 1048-1058, 2010.

13. Hara T, Nakamura K, Matsui M, et al: Suppression of basal autophagy in neural cells causes neurodegenerative disease in mice. Nature 441: 885-889, 2006.

14. Komatsu M, Waguri S, Chiba T, et al: Loss of autophagy in the central nervous system causes neurodegeneration in mice. Nature 441: 880-884, 2006.
15. Sarkar S, Ravikumar B, Floto RA and Rubinsztein DC: Rapamycin and mTOR-independent autophagy inducers ameliorate toxicity of polyglutamine-expanded huntingtin and related proteinopathies. Cell Death Differ 16: 46-56, 2009.

16. Pan T, Kondo S, Zhu W, Xie W, Jankovic J and Le W: Neuroprotection of rapamycin in lactacystin-induced neurodegeneration via autophagy enhancement. Neurobiol Dis 32: 16-25, 2008.

17. Wong E and Cuervo AM: Autophagy gone awry in neurodegenerative diseases. Nat Neurosci 13: 805-811, 2010.

18. Keane RW, Kraydieh S, Lotocki G, et al: Apoptotic and anti-apoptotic mechanisms following spinal cord injury. J Neuropathol Exp Neurol 60: 422-429, 2001.

19. Yoshino O, Matsuno H, Nakamura H, et al: The role of Fas-mediated apoptosis after traumatic spinal cord injury. Spine (Phila Pa 1976) 29: 1394-1404, 2004.

20. Casha S, Yu WR and Fehlings MG: FAS deficiency reduces apoptosis, spares axons and improves function after spinal cord injury. Exp Neurol 196: 390-400, 2005.

21. Erlich S, Alexandrovich A,Shohami Eand Pinkas-KramarskiR: Rapamycin is a neuroprotective treatment for traumatic brain injury. Neurobiol Dis 26: 86-93, 2007.

22. Sekiguchi A, Kanno H, Ozawa H, Yamaya S and Itoi E: Rapamycin promotes autophagy and reduces neural tissue damage and locomotor impairment after spinal cord injury in mice. J Neurotrauma 29: 946-956, 2012.

23. Duran A, Serrano M, Leitges M, et al: The atypical PKC-interacting protein p 62 is an important mediator of RANK-activated osteoclastogenesis. Dev Cell 6: 303-309, 2004.

24. Rodriguez A, Durán A, Selloum M, et al: Mature-onset obesity and insulin resistance in mice deficient in the signaling adapter p62. Cell Metab 3: 211-222, 2006.

25. Duran A, Linares JF, Galvez AS, et al: The signaling adaptor p62 is an important NF-kappaB mediator in tumorigenesis. Cancer Cell 13: 343-354, 2008.

26. Kuusisto E, Salminen A and Alafuzoff I: Ubiquitin-binding protein p62 is present in neuronal and glial inclusions in human tauopathies and synucleinopathies. Neuroreport 12: 2085-2090, 2001.

27. Zatloukal K, Stumptner C, Fuchsbichler A, et al: p62 is a common component of cytoplasmic inclusions in protein aggregation diseases. Am J Pathol 160: 255-263, 2002.

28. Nagaoka U, Kim K, Jana NR, et al: Increased expression of p62 in expanded polyglutamine-expressing cells and its association with polyglutamine inclusions. J Neurochem 91: 57-68, 2004.

29. Komatsu M, Waguri S, Koike M, et al: Homeostatic levels of p62 control cytoplasmic inclusion body formation in autophagy-deficient mice. Cell 131: 1149-1163, 2007.

30. Jin Z, Li Y, Pitti R, et al: Cullin3-based polyubiquitination and p62-dependent aggregation of caspase- 8 mediate extrinsic apoptosis signaling. Cell 137: 721-735, 2009.

31. Sanz L, Diaz-Meco MT, Nakano H and Moscat J: The atypical PKC-interacting protein $\mathrm{p} 62$ channels NF-kappaB activation by the IL-1-TRAF6 pathway. EMBO J 19: 1576-1586, 2000.

32. Moscat J, Diaz-Meco MT, Albert A and Campuzano S: Cell signaling and function organized by PB1 domain interactions. Mol Cell 23: 631-640, 2006.

33. Moscat J and Diaz-Meco MT: p62 at the crossroads of autophagy, apoptosis, and cancer. Cell 137: 1001-1004, 2009.

34. Xu P, Gong WM, Li Y, et al: Destructive pathological changes in the rat spinal cord due to chronic mechanical compression. Laboratory investigation. J Neurosurg Spine 8: 279-285, 2008.

35. Basso DM, Beattie MS and Bresnahan JC: A sensitive and reliable locomotor rating scale for open field testing in rats. J Neurotrauma 12: 1-21, 1995.

36. Kuusisto E, Kauppinen T and Alafuzoff I: Use of p62/SQSTM1 antibodies for neuropathological diagnosis. Neuropathol Appl Neurobiol 34: 169-180, 2008.

37. Karin M and Lin A: NF-kappaB at the crossroads of life and death. Nat Immunol 3: 221-227, 2002.

38. Sheikh MS and Huang Y: Death receptor activation complexes: it takes two to activate TNF receptor 1. Cell Cycle 2: 550-552, 2003. 